\title{
Computational High-throughput Screening for Solar Energy Materials
}

\section{Castelli, Ivano Eligio; Thygesen, Kristian Sommer; Jacobsen, Karsten Wedel}

Published in:

Theoretical Modeling of Organohalide Perovskites for Photovoltaic Applications

Publication date:

2017

Document Version

Peer reviewed version

Link back to DTU Orbit

Citation (APA):

Castelli, I. E., Thygesen, K. S., \& Jacobsen, K. W. (2017). Computational High-throughput Screening for Solar Energy Materials. In Theoretical Modeling of Organohalide Perovskites for Photovoltaic Applications CRC Press.

\section{General rights}

Copyright and moral rights for the publications made accessible in the public portal are retained by the authors and/or other copyright owners and it is a condition of accessing publications that users recognise and abide by the legal requirements associated with these rights.

- Users may download and print one copy of any publication from the public portal for the purpose of private study or research.

- You may not further distribute the material or use it for any profit-making activity or commercial gain

- You may freely distribute the URL identifying the publication in the public portal

If you believe that this document breaches copyright please contact us providing details, and we will remove access to the work immediately and investigate your claim. 


\title{
Computational High-throughput Screening for Solar Energy Materials
}

\author{
Ivano E. Castelli, ${ }^{* \dagger}$ Kristian S. Thygesen, ${ }^{*}$ and Karsten W. Jacobsen*
}

December 22, 2015

The design and development of new materials is required to meet the challenge of sustainable and environmental friendly energy production and storage. Computer simulations in the form of atomic-scale electronic structure calculations can be expected to play an important role in this as demonstrated over the last decade. Most of such calculations are carried out in the framework of Density Functional Theory (DFT) $[1,2]$ which demonstrates a good compromise between computational speed and accuracy of the calculations. The ever-increasing computational power makes it possible today to study fairly complex structures with several hundreds or even thousands of atoms. A few examples of materials design studies include the search for novel materials for carbon capture and storage, [3] batteries, [4] stable binary and ternary alloys, [5] transparent conductors, [6] dye sensitized solar cells, [7] photovoltaics, [8, 9] and water splitting materials. [10, 11, 12]

The calculations usually address only a small part of the actual design problem and is nowhere near direct simulations of the functioning material. An important issue is therefore to determine a few simple parameters, so-called descriptors or design metrics, which describe key properties for the material func-

${ }^{*}$ Center for Atomic-scale Materials Design, Department of Physics, Technical University of Denmark, DK 2800, Kongens Lyngby, Denmark.

${ }^{\dagger}$ Present address: Department of Chemistry, University of Copenhagen, DK 2100, Copenhagen $\varnothing$, Denmark. E-mail: ivano@chem.ku.dk 
tion and which at the same time can be computed at the atomistic level. [13] For example, the formation enthalpy is a possible descriptor for the stability of a compound, the band gap for its absorption properties, and the binding energies of reaction intermediates for the catalytic activity, [14] and so on.

In this chapter, we first present some descriptors useful for identifying novel semiconductors for light harvesting in a photovoltaic or photoelectrocatalytic

cell, and afterwards, we review the application of a screening procedure to more than 20000 inorganic perovskites in the cubic and layered phases. Furthermore, we study trends and propose a handful of materials for further investigation. Lastly, we report the trends for a smaller set of 300 hybrid halide perovskites. Our ab-initio quantum mechanics simulations have been performed in the framework of DFT, using the GPAW code. [15, 16] All our results are collected in the open source and open data Computational Materials Repository (CMR) database. [17]

\section{Identification of Descriptors}

To harvest and convert solar light into electrical energy in photovoltaic (PV) cells or into chemical energy using photoelectrochemical (PEC) reactions require as the first step the absorption of light creating electron-hole pairs. The absorption is performed by a semiconducting material which has to be optimized for efficient absorption and which allows for further transformation of the energy in the electron-hole pairs.

A list of some - but certainly not all - properties that a semiconducting material has to fulfill to be used in a PEC cell might include (i) chemical and structural stability, (ii) good light absorption, (iii) photogenerated charges at the correct potentials and with good mobility, (iv) low cost and non-toxicity. Stability, good light absorption, and low cost are also among the desired prop- 
erties for a materials to be used in a PV device. The PEC technology is more challenging than the one behind PV cells because the electron and hole transfer should induce chemical reactions. However, the benefit is that high energy storage capacity is available in the chemical bonds of the produced fuels.

There are many other issues that have to be dealt with in a working PEC device. One of the most important ones is the catalysis of the chemical processes happening at the material surfaces. The light absorbing materials should therefore either have good catalytic properties themselves or be able to interface both structurally and electronically to efficient catalysts. [18] However, none of these issues shall be addressed here.

In the following we shall discuss the computational descriptors that are used in the screening studies. The descriptor for the stability is the heat of formation for the material or more specifically the calculated total energy difference between the material and competing structures and materials at zero temperature. The simplest measure is the standard heat of formation which is calculated as the energy difference between the material at hand and its components in their standard states. However, we also go beyond this by not only considering phase separation into elemental materials but also into a pool of reference materials of varying compositions and structures. The pool includes more than 2000 structures from the ICSD [19] and the Materials Project database. [20] If the energy of the candidate material is above the calculated convex hull (plus a metastability region of $0.2 \mathrm{eV} /$ atom) that define the stability frontier, the material under investigation is considered unstable. The total energies are calculated using the RPBE as exchange-correlation functional in the generalized gradient approximation (GGA). [21]

If the material is in contact with water, as, for example, in a PEC device without protection layers of the light absorber, the stability against dissolution 
should also be considered. The stability in water can be calculated by means of Pourbaix diagrams. A Pourbaix diagram is a pH-potential phase diagram which contains both solid and dissolved species in the pool of reference systems. In our case, the energy of the solid phases are calculated by DFT as above and the dissolution energies are obtained from experimental results. [22, 23] Although Pourbaix diagrams give information about the bulk thermodynamical stability in water of a compound, they do not provide details about the reaction kinetics and the possible role of surfaces and their passivation.

The absorption properties of a material can be described, to a first approximation, by the band gap, assuming that photons with energies above the gap are absorbed while lower energy photons are not. Within DFT, the simplest estimate of the band gap would be the calculated gap in the single-particle Kohn-Sham spectrum. However, this leads to severe underestimation of the gap because of the so-called derivative discontinuity [24] and the self-interaction error for semi-local exchange-correlation functionals. [25] Several methods have been proposed to take these effects into account, usually at a significantly higher computational cost. Hybrid functionals like PBE0 or HSE06, which include a fraction of exact exchange, or many-body methods such as the GW approximation improve the estimation of the band gaps, but are considerably more computationally expensive than the semi-local functionals.

As a compromise we calculate the band gaps using an improved description of the so-called GLLB functional, [26] called GLLB-SC, [27] particularly suited for solids. This functional is an additional approximation to the KLI approximation $[28,29]$ to the exact exchange optimized effective potential (EXX-OEP). [30] As a rather unique feature, it includes an explicit evaluation of the derivative discontinuity.

We note that the gap discussed above is the so-called quasi-particle (QP) 
or fundamental gap, which is the difference between the first ionization potentials (IP) and the electron affinity (EA). It can be directly measured by photo-emission and inverse photo-emission experiments and within DFT it is obtained computationally by adding the derivative discontinuity, $\Delta_{\mathrm{xc}}$, to the Kohn-Sham (KS) gap: $E_{\text {gap }}^{\mathrm{QP}}=I P-E A=E_{\text {gap }}^{\mathrm{KS}}+\Delta_{\mathrm{xc}} \cdot \mathrm{QP}$ gaps do not include excitonic effects which can result in a lower optical band gap. However, we shall not go any further into this issue here.

The GLLB-SC calculated band gaps show an error of the order $0.5 \mathrm{eV}$ when tested against non-self-consistent $\mathrm{G}_{0} \mathrm{~W}_{0}$ values and experiments for single metal oxides, [10] binary semiconductors, [31] and for oxo-perovskites with a band gap in the visible range. [32] More recently, we have benchmarked the GLLB-SC against different levels of the $\mathrm{GW}$ approximation $\left(\mathrm{G}_{0} \mathrm{~W}_{0}, \mathrm{GW}_{0}\right.$, and $\left.\mathrm{GW}\right)[33]$ and the hybrid HSE06 [34] for a set of 20 ternary and quaternary semiconductors. [35] Among the considered methods, the GLLB-SC gives the best approximation to the eigenvalue-self-consistent $\mathrm{GW}$, with a mean relative error of around $15 \%$. We therefore expect that the GLLB-SC is accurate enough for high-throughput calculations since it provides reliable results at only a slight increase in computational cost relative to standard GGA calculations even for large crystal structures.

An appropriate band gap is certainly a requirement for achieving efficient visible light absorption, however, it does not take into account the actual strength of the absorption at different photon energies. The absorption might for example be limited by symmetry or by lack of spatial overlap between electronic states in the valence and conduction bands. Therefore we in some cases perform explicit calculation of the absorption spectrum within time-dependent DFT where both the character of the band gap (direct/indirect) and the strengths of the dipoletransition matrix elements are included. [36] We note that in screening studies 
by Yu and Zunger [37] the band gap characters and the absorption strengths have been combined into a single descriptor or metric.

In a PEC device, the photogenerated charges have to be at the right potential with respect to the red-ox levels of water to be able to induce the water oxidation and the proton reduction reactions. We use a simple empirical approach $[38,39]$ where the positions of the electron and hole levels relative to vacuum for a material with $N$ atoms in the unit cell is estimated by first calculating the center of the gap as the geometrical average of the electronegativities, $X_{i}$, of the constituent elements, $i$, with the Mulliken's scale. [40] The conduction and valence band edges with respect to the vacuum level, $E_{\mathrm{VB}}$ and $E_{\mathrm{CB}}$, are then obtained by adding and subtracting half of the gap, respectively:

$E_{\mathrm{CB}, \mathrm{VB}}=\left(\prod_{i} X_{i}\right)^{1 / N} \pm E_{\mathrm{gap}} / 2$. More sophisticated models, which include the investigation of surface properties and of realistic electrochemical environments, can be also used but at increased computational cost. [41, 42]

Low cost and non-toxicity of a material are also desirable properties and can at least to some extent be estimated based on the constituent elements. The cost can for example further be related to the abundance of the element. [43] However, in the following screening studies we do not exclude elements based on their abundance or cost.

\section{Inorganic Perovskites}

In the following we consider compounds in the cubic perovskite structure with the formula $\mathrm{ABO}_{3}$ (space group $\mathrm{Pm} \overline{3} \mathrm{~m}$ ). We select this structure because of the generally high stability and because of the large variety of properties and applications of materials with this structure. [44] The unit cell of the cubic perovskite is formed of 5 atoms (two inequivalent metals, a large 12-coordinated cation (A-ion) and a small 6-coordinated cation (B-ion) and three non-metals) 


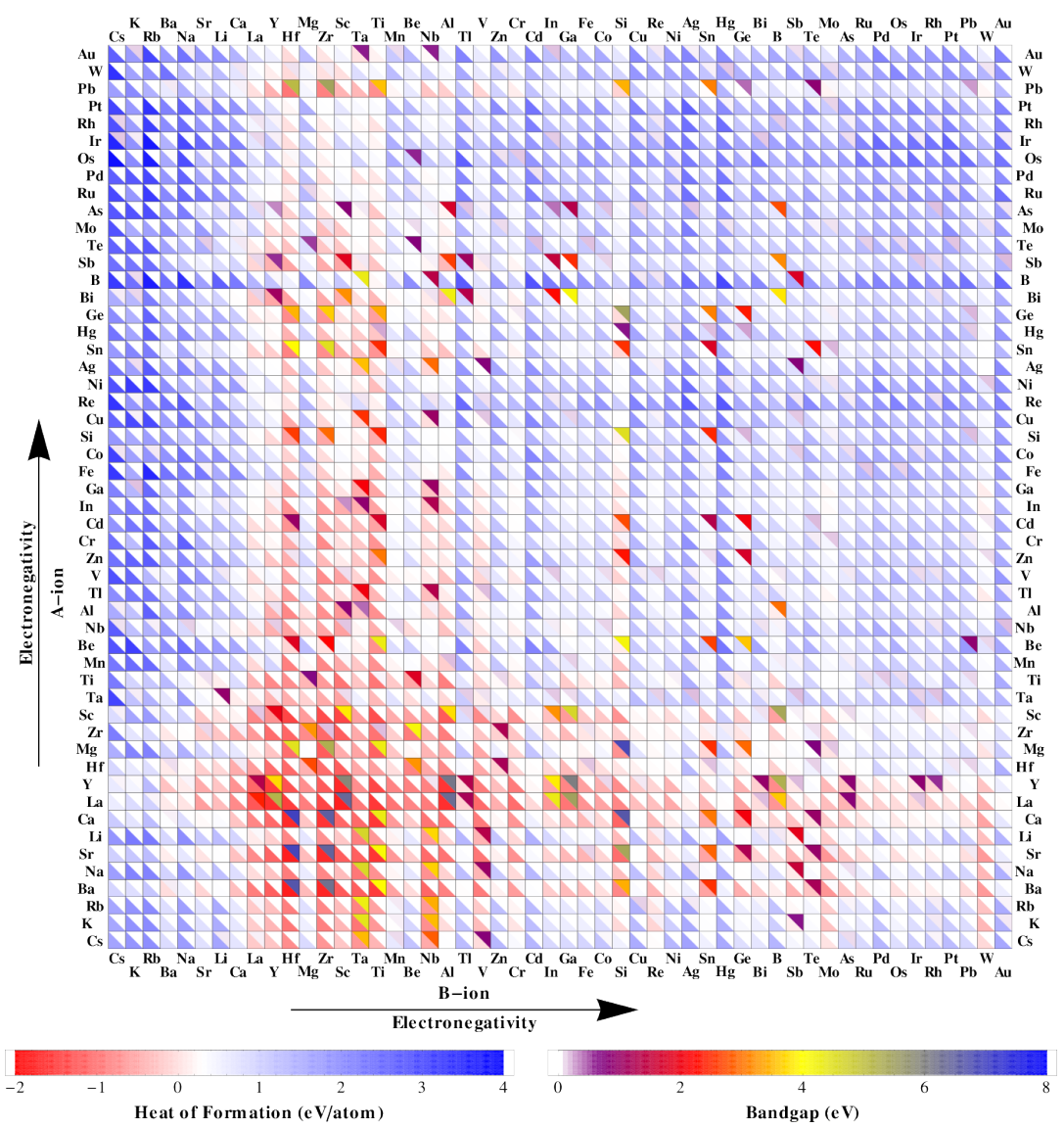

Figure 1: Calculated heats of formation per atom and band gaps of perovskite binary metal oxides. In each square the formation energy (lower, left triangle) and the band gap (upper, right triangle) of an oxide are shown. The interesting combinations for water splitting are indicated with red squares. The chemicals are sorted for increasing electronegativity. Figure from Ref. [10]

and it can accommodate most of the elements in the periodic table.

First we consider all the possible cubic perovskites obtained by combining 52 non-radioactive metals of the periodic table in the A and B sites. As non-metals we start out with oxygen, because of the high stability of oxides.

Figure 1 shows the formation energies per atom and the band gaps for the 2704 oxides. Each square which corresponds to a particular oxide containing 
two metals is divided into two triangles where the lower left one indicates the stability (from red to blue with decreasing stability) and the upper right one, the band gap. It is apparent from the figure that there is a clear trend in the heat of formation where low electronegativity of the constituent metal atoms favors stability. The band gap does not show such a clear trend, however, there is a certain tendency that the band gaps increase with a decrease of the Bion electronegativity or with a decrease of the crystallographic symmetry be adjusting the size of the A-ion. [45]

The calculated data show that most of the semiconducting compounds have too large a band gap, and that the band edges are too deep with respect to the redox levels of water. We therefore also investigate other anions in particular nitrogen because the the $\mathrm{N} 2 p$ levels are placed higher in energy than the $\mathrm{O}$ $2 p$ levels and since the $p$-states dominate the valence band edge, smaller band gaps and in fact also a better matching with the redox levels of water can be obtained. However, the nitrides generally are less stable than the oxides.

Including nitrogen, sulfur and fluorine as possible anions in the cubic perovskite structure we have established a database of cubic perovskites with almost 19000 combinations of metal atoms and anions. [10, 11]

It is possible to "mine" the database to discover trends and correlations between the materials for example by applying clustering algorithms and construction of dendrograms. [46] The analysis shows what influence the stability of the compounds and their ability to form band gaps. Three fairly general rules is found to apply: (i) The number of electrons in the supercell has to be even. Without this a non-magnetic material will have an electronic band crossing the Fermi level and therefore be metallic. (ii) The sum of the possible oxidation numbers has to be zero. Again otherwise a band gap cannot be formed. (iii) The atom in the A-site should have a larger radius than the atom in the B- 


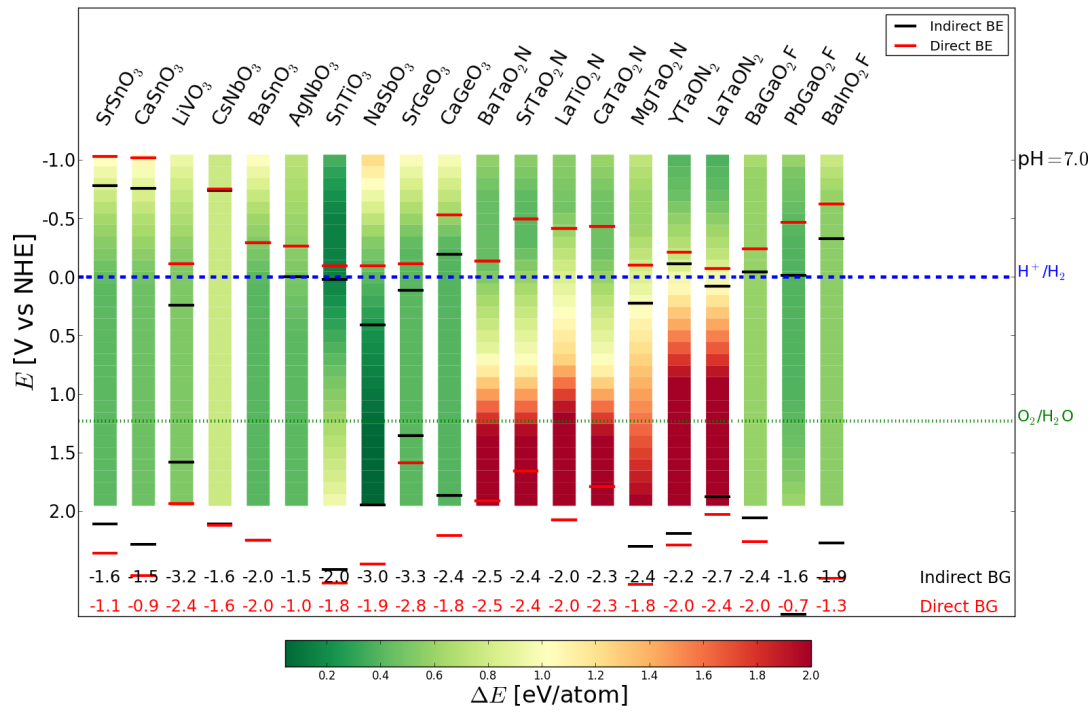

Figure 2: Identified perovskites with potential for one-photon water splitting. The stability in water of each material is estimated as the energy difference between the material and the most stable phases (solid and aqueous) in which it can separate in a potential range between -1 and $2 \mathrm{~V}$ and at $\mathrm{pH}=7$. The color scale runs from green (stable) to red (unstable compounds). The indirect and direct positions of the valence and conduction band edges (BE) are indicated in black and red as well as the indirect and direct band gap (BG) and the levels for hydrogen and oxygen evolution.

site. This rule is in spirit similar to rules based on the Goldschmidt's tolerance factor, but turns out to be much better fulfilled. Applying these rules to for example layered perovskites leads to a significant speed-up in the identification of relevant materials. [32]

Returning to the database of cubic perovskites, it can be taken as a starting point for identifying materials of relevance for water splitting by applying the following three screening criteria. (i) Heat of formation relative to the pool of reference systems of less than $0.2 \mathrm{eV} /$ atom to ensure stability. (ii) Band gap in the visible range between $1.5 \mathrm{eV}$ and $3.0 \mathrm{eV}$. The lower limit accounts for the bare energy to run the water-splitting reaction $1.23 \mathrm{eV}$ plus $\sim 0.25 \mathrm{eV}$ to account for the electrochemical overpotentials of hydrogen and oxygen. [47] This 
limit may be higher if the splitting of the quasi-Fermi levels is smaller than the gap when the SC is under illumination. [48] If the gap is above $3.0 \mathrm{eV}$ too little of the solar spectrum is absorbed. Depending on construction of the device, the photoactive material in a cell may be thin or thick and we therefore perform the search for either the direct or the indirect gap. (iii) Without considering the overpotentials, the valence and conduction band edges should at least fulfill $\mathrm{VB}_{\text {edge }}>1.23 \mathrm{~V}$ vs the Normal Hydrogen Electrode (NHE) and $\mathrm{CB}_{\text {edge }}<0 \mathrm{~V}$ vs NHE for appropriate alignment of the band edges with the water redox levels.

Out of the 19000 compounds in the database only 20 fulfill these criteria: 10 oxides, 7 oxynitrides, and 3 oxyfluorides as shown in Figure 2. A further investigation of the crystal structure of these combinations show that $\mathrm{SrSnO}_{3}$ and $\mathrm{CaSnO}_{3}$ undergo lattice distortions and in their most stable structure, they have gaps beyond the visible light absorption limit. $\mathrm{AgNbO}_{3}$ and $\mathrm{BaSnO}_{3}$ as well as the oxynitride series $\left(\mathrm{BaTaO}_{2} \mathrm{~N}, \mathrm{SrTaO}_{2} \mathrm{~N}, \mathrm{CaTaO}_{2} \mathrm{~N}, \mathrm{LaTiO}_{2} \mathrm{~N}\right.$, and $\mathrm{LaTaON}_{2}$ ) have been tested as water splitting materials. Due to defect-assisted recombination, $\mathrm{BaSnO}_{3}$ shows no activity, [49], while $\mathrm{AgNbO}_{3}$ splits water in visible light in the presence of sacrificial reagents, [50] and the oxynitrides perform well for hydrogen evolution. [51] However, the oxynitrides may have stability issues in contact with water at high potentials as also seen in Figure 2 To our knowledge no other cubic perovskites that can split water in visible light have been identified. In addition to this set, we identify materials, like $\mathrm{AgTaO}_{3}$ and $\mathrm{SrTiO}_{3}$, who can split water under UV light.

As mentioned earlier the band gap is only a rough descriptor of the energy conversion efficiency. A more accurate estimation of the absorbed light can be obtained by considering the full absorption spectrum as calculated within timedependent DFT. Details of the approach can be found in Ref. [36]. The results of such a calculation for the 20 perovskites identified above are shown in Fig. 3. 


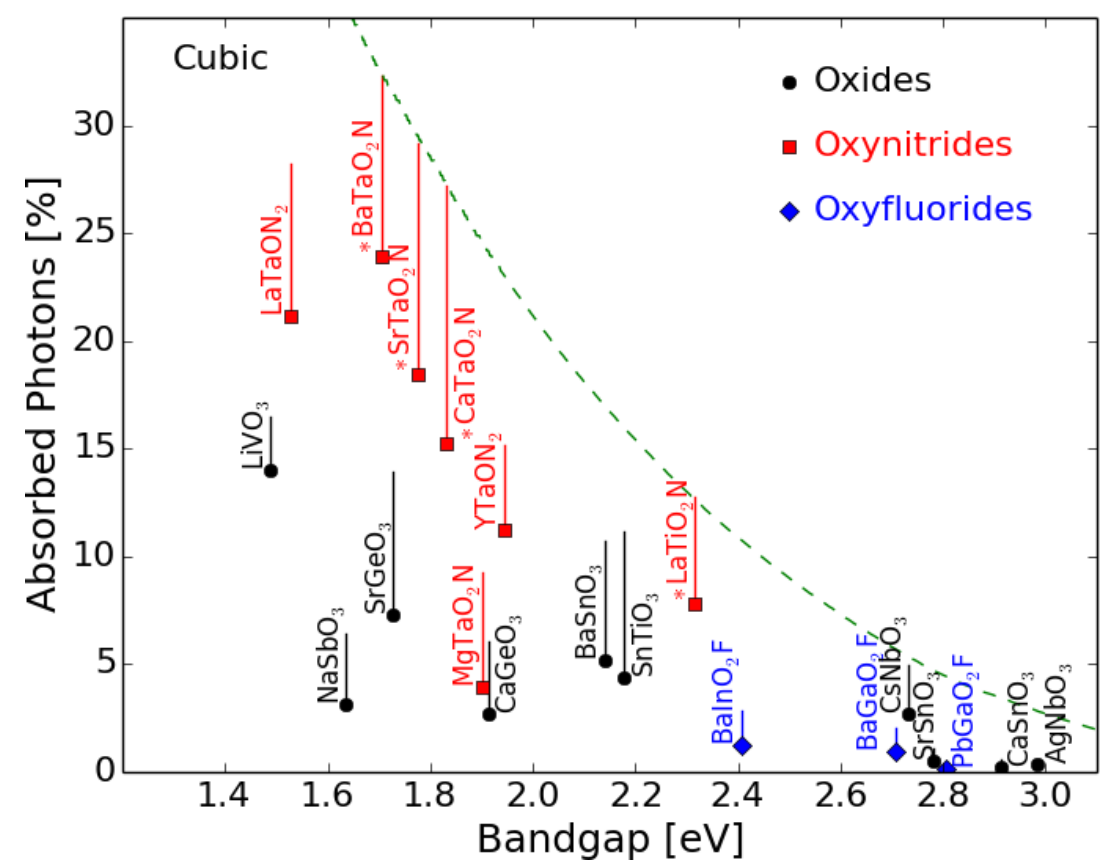

Figure 3: Light harvesting efficiency of the cubic perovskites of Figure 2. The markers indicate the efficiency of a $100 \mathrm{~nm}$-thick material, while the vertical lines go up to the limit of infinite thickness. The green dashed line indicates the maximum theoretical efficiency as a function of the (possibly indirect) band gap. The materials marked with ${ }^{*}$ have a direct transition at the band gap. Figure from Ref. [52]

The absorption efficiencies are estimated as the ratio between the absorbed and the total amount of photons from the sun at AM1.5. In Figure 3, the efficiencies are plotted as a function of the possibly indirect band gap for a material thickness of $100 \mathrm{~nm}$. The vertical lines go up to the result for infinite thickness corresponding to absorption of all light above the direct band gap if the matrix element at the band edge is non-vanishing. All the oxides and oxyfluorides have small efficiencies due to at least two factors: they have indirect band gaps (from the R-point, with the wave functions usually located on the $2 p$-levels of oxygen, to the $\Gamma$-point, with wave functions localized on the $s$ - or $d$-levels of the B-ion) and the absorption of photons for thin films therefore start 
at a higher energy corresponding to at least the indirect band gap. Also the dipole transition matrix elements are rather small due to the lack of overlap between the wave functions at the band edges. The absorption at the onset is therefore not very intense. Only $\mathrm{LiVO}_{3}$ has an efficiency which gets close to $15 \%$ for a $100 \mathrm{~nm}$-thick material. All the others are below 10\%, and in all these cases, the band gap is also a fairly poor descriptor of the efficiencies.

The oxynitrides, instead, show a higher efficiency: most of them have direct band gaps (at the $\Gamma$-point, with the states at the valence band maximum dominated by the nitrogen $2 p$ states with a small hybridization from the oxygen $2 p$-levels and the states at the conduction band minimum composed by the $d$-levels of the B-ion) with a more intense absorption at the offset. Since the transition at the band gap is allowed, these materials have the maximum theoretical efficiency allowed by the gap, in the limit of infinite thickness. [52]

So in summary, the perovskite oxides and oxyfluorides are seen to possess indirect band gaps with fairly low absorption while some of the oxynitrides have both direct gap and more efficient absorption. The band edges of the oxynitrides are also generally positioned better for the water splitting reaction. [51] However, as also pointed out before, the oxynitrides are not so stable, which can be traced to breaking of the symmetry of the octahedra. The stability is particularly reduced in contact with water at high electron potential.

The computational approach discussed here has also been applied to the screening of different lower symmetry perovskites like layered or double perovskites aimed at one- and two-photon water splitting or at identification of protecting shields against dissolution. [32, 52, 53] 


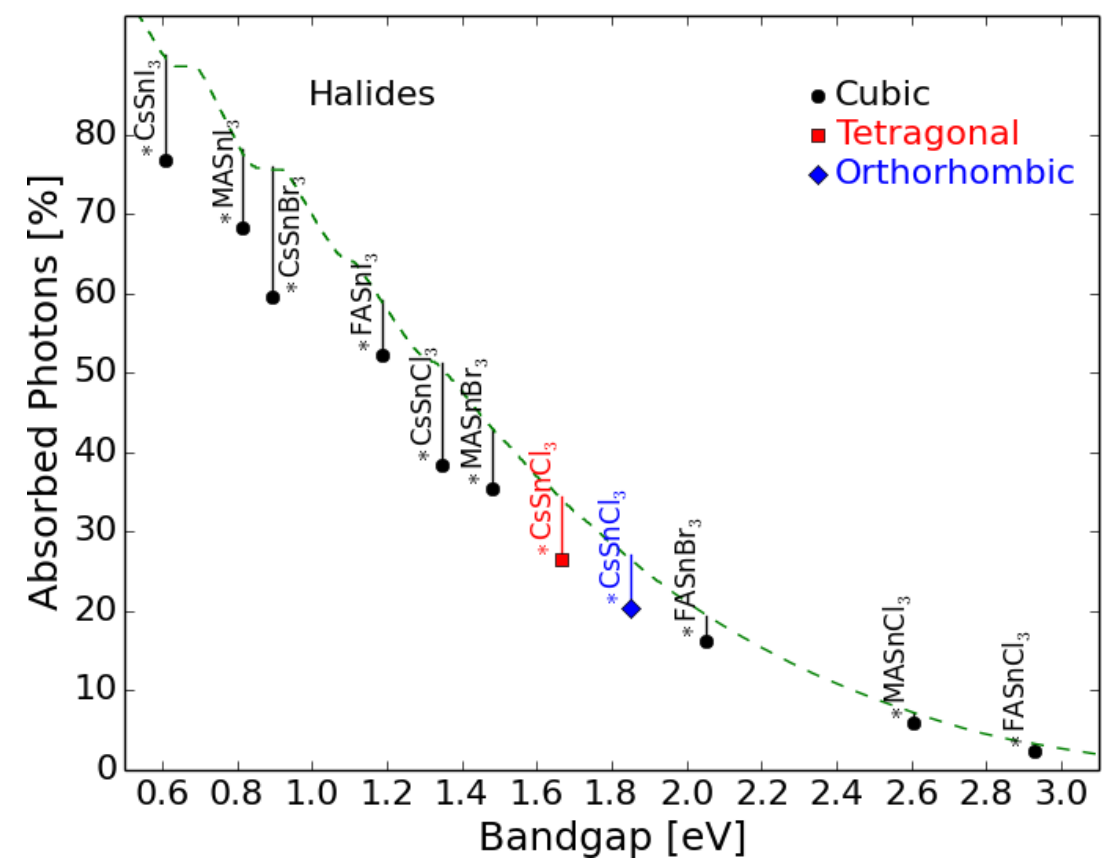

Figure 4: Light harvesting efficiency for hybrid halide perovskites in different symmetries (cubic: black dots, tetragonal: red square, and orthorhombic: blue diamond). Figure from Ref. [52]

\section{$3 \quad$ Hybrid Halide Perovskites}

Over the past few years new hybrid halide perovskites have attracted immense interest as PV light absorbers starting with studies of $\mathrm{CH}_{3} \mathrm{NH}_{3} \mathrm{PbBr}_{3}$ and $\mathrm{H}_{3} \mathrm{NH}_{3} \mathrm{PbI}_{3}$ [54] and followed by numerous other investigations of these and related systems. $[55,56,57,58]$

The screening approach discussed in the previous section can also be applied to the class of hybrid halide perovskites. In the following we shall more specifically report investigations of perovskites obtained by combining cesium $(\mathrm{Cs})$, methylammonium $\left(\mathrm{MA}, \mathrm{CH}_{3} \mathrm{NH}_{3}{ }^{+}\right.$), or formamidinium (FA, $\mathrm{HC}\left(\mathrm{NH}_{2}\right)_{2}{ }^{+}$) as A-ion, tin $\left(\mathrm{Sn}^{++}\right)$or lead $\left(\mathrm{Pb}^{++}\right)$as B-ion, and chlorine $\left(\mathrm{Cl}^{-}\right)$, bromine $\left(\mathrm{Br}^{-}\right)$, iodine $\left(\mathrm{I}^{-}\right)$, or their combinations as anions. We also allow for four differ- 
ent symmetries (cubic, tetragonal, and two orthorhombic phases with space groups $\mathrm{P}_{\mathrm{m} \overline{3} \mathrm{~m}}, \mathrm{P}_{4 / \mathrm{mbm}}, \mathrm{P}_{\mathrm{bnm}}$, and $\mathrm{P}_{\mathrm{nma}}$, respectively) for a total of 240 combinations [59] which have been fully relaxed using the PBEsol exchange-correlation functional. [60]

Figure 4 shows the light absorption efficiencies for a selection of hybrid halide perovskites analogous to Figure 3 for the oxides, oxynitrides, and oxyfluorides. The superior absorption properties of the halide perovskites are immediately apparent. They exhibit a direct band gap transition with the states at the valence band maximum located on the tin/lead $s$-states and the conduction band minimum on the tin/lead $p$-states. This leads to a strong overlap with a large dipole matrix elements.

The stability of the perovskites in the four different phases have been investigated in Ref. [59] At low temperatures for all materials, the cubic phase is less stable than the lower symmetry phases (tetragonal and both of the orthorombic phases) which come into play depending on the detailed composition. However, the energy difference between the different phases turns out to be small typically below $0.1 \mathrm{eV}$ per formula unit. Mixing the halide atoms typically cost energy, except for the compound $\mathrm{FASnBr}_{2} \mathrm{I}$, which turn out to be marginally more stable than the corresponding bromide and iodide. However, again the energy differences are small and the combined system can be expected to be metastable considering also that the entropy of mixing entropy is not taken into account. [61] In fact, both Cs and MA mixed compounds have already been synthesized. [58, 62]

The high absorption efficiency with direct gaps and strong dipole matrix elements independent of the detailed composition (Figure 4) makes it of interest to study the variation of the band gap in the whole database of 240 compounds. Due to the orbitals involved in the transition at the band gap, the spin-orbit 


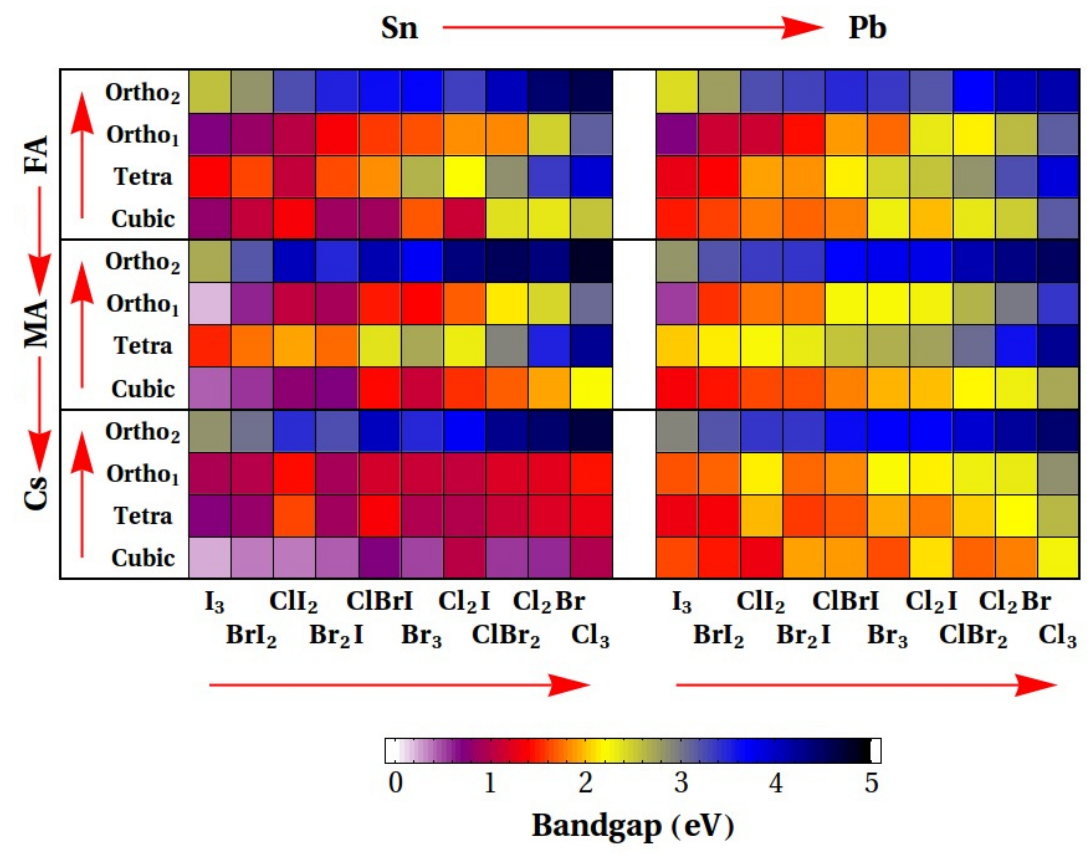

Figure 5: The 240 calculated gaps. Each square corresponds to a different phase and composition. The phase and the A-ion are indicated by the labels on the left vertical axis. The B-ion and the anion composition are shown on the top and bottom of the horizontal axis, respectively. The anion combinations are sorted according to increased geometrical mean of the electronegativity. The increase of the gaps with respect to a change in the phase and composition is followed by red arrows. Figure from Ref. [59]

effect plays an important role in the calculation of the band gaps, in particular in the lead-based perovskites for which the effect is larger. $[63,64]$ Test calculations on a limited set of systems show that the spin-orbit correction, $\Delta_{\text {soc }}$, is rather independent of the A-ion and anion configuration but depends sensitively on the character of the B-ion. Based on the calculations on the test systems we therefore introduce an $a d-h o c$ reduction of the band gap of $0.25 \pm 0.05 \mathrm{eV}$ for the Sn perovskites and of $1.02 \pm 0.06 \mathrm{eV}$ for the $\mathrm{Pb}$ systems. Focusing on the optical band gap we also need to take into account the exciton energies which based on calculations using the Bethe-Salpeter Equation are found to lead to a 
reduction of the gap in a range from 0.11 to $0.15 \mathrm{eV}$ for all the cubic systems formed by $\mathrm{Cs}$ with $\mathrm{I}_{3}, \mathrm{Br}_{3}$, or $\mathrm{Cl}_{3}$. We thus reduce the calculated gaps by $\Delta_{\mathrm{e}-\mathrm{h}}=0.13 \mathrm{eV}$ to take this effect into account. Summarizing, the optical gap is therefore obtained starting from the GLLB-SC Kohn-Sham band gap and adding the derivative discontinuity contribution, which depends on the composition and phase considered, and subtracting the spin-orbit and electron-hole interaction corrections. The agreement between the calculated and measured gaps for the cases where experimental studies have been carried out is very good, with a mean absolute error of $0.2 \mathrm{eV}$. [59]

In Figure 5, we report the calculated optical band gaps of the 240 systems. Each colored square represents a particular phase and composition. The band gaps span over a region between 0.5 and $5 \mathrm{eV}$. We can identify several trends, summarized in the figure by red arrows, which can be helpful to design novel materials with gaps in a desired region. We note: (i) The band gaps increase as the size of the A-cation is decreased. The FA molecule is larger than MA which is again larger than the $\mathrm{Cs}$ ion. The reason behind this effect is that a larger cation leads to a larger lattice constant which again leads to a down-shift of the $\mathrm{Sn}$ or $\mathrm{Pb} s$-states at the valence band maximum. [65, 66] (ii) The $\mathrm{Pb}$ systems have a larger gap than the Sn systems. (iii) The band gaps increase with increased electronegativity of the anions. This is clearly seen in Figure 5 where the anions are listed according to this parameter. This effect is probably caused by the more electronegative anions pulling down the extended $\mathrm{Sn}$ or $\mathrm{Pb}$ $s$-states more than the more localized $p$-states. (iv) Symmetry breaking leads generally to increased band gaps so that the cubic phase exhibit smaller gaps than the other phases. In particular the orthorhombic 2 phase exhibit higher gaps than the other phases.

The character of the A-cation may play an important role for the function- 


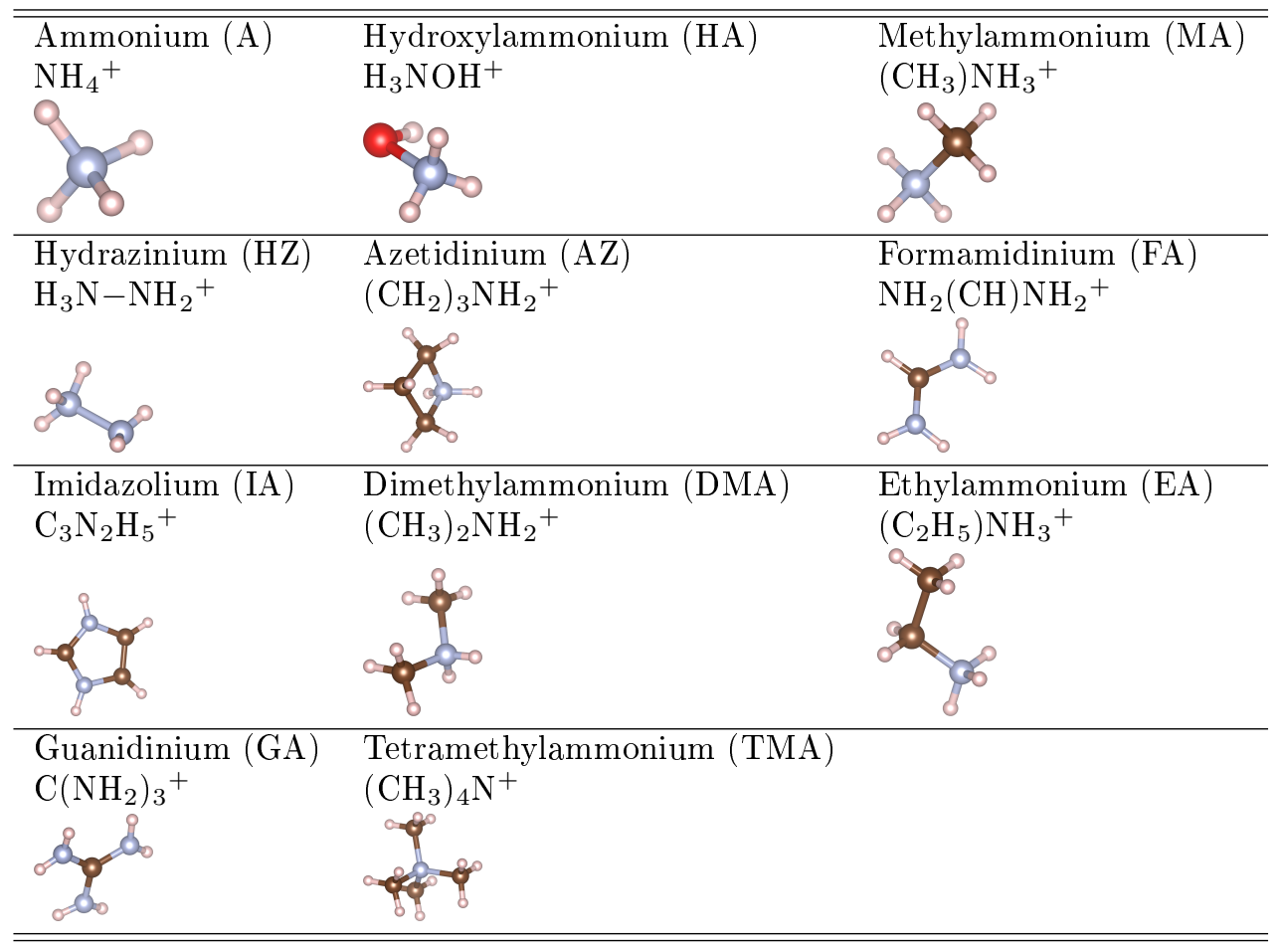

Table 1: Organic molecules investigated as A-cation. [67] 
ality of a hybrid halide perovskite as PV material. It has for example been suggested that the spontaneous electric polarization of A-cation molecules is of key importance. [68] We therefore in the following investigate the properties of perovskites with different common nitrogen based cations (Table 1) together with $\mathrm{Sn}($ or $\mathrm{Pb})$ and $\mathrm{I}_{3}$ in the cubic phase. For each structure we generate 10 different configurations where the molecule is randomly rotated. Each structure is then allowed to relax, in both lattice and atomic positions, in the 5 atoms unit cell. Even though, the 5 atom unit cell is not large enough to encompass all the possible distortions, it gives an estimate of the coupling between the molecule orientation, volume and lattice distortions, and their effects on the magnitude of the band gap.

In Figure 6, the calculated band gaps for the systems $\mathrm{MSnI}_{3}$ (or $\mathrm{MPbI}_{3}$, where $\mathrm{M}$ is one of the organic molecules) are plotted as a function of the pseudocubic lattice parameter, defined as the cubic root of the unit cell volume. The error bars along the $x$-axis indicate the average non-cubicity, defined as the average of $\frac{1}{3} \sum_{i=x, y, z}\left|a_{i}-a_{\mathrm{pc}}\right|$, where $a_{i}$ is the lattice parameter along the $i$ 'th direction and $a_{\mathrm{pc}}$ is the pseudo-cubic lattice parameter. The error bars on the gap axis represent the variation of the gap depending on the orientation of the molecules.

The Sn-based systems show an almost linear relation between the pseudocubic lattice parameter and the band gap, and the molecules are clearly ordered according to size. This confirms the gap-volume trend discussed earlier, and opens up for significant band gap modification by molecular replacement. The correlation is less clear for the $\mathrm{Pb}$-based systems but again the molecules are roughly ordered by size.

For the smaller molecules the lattice distortions are seen to be less pronounced than for the larger ones pointing to a - maybe not unexpected - 


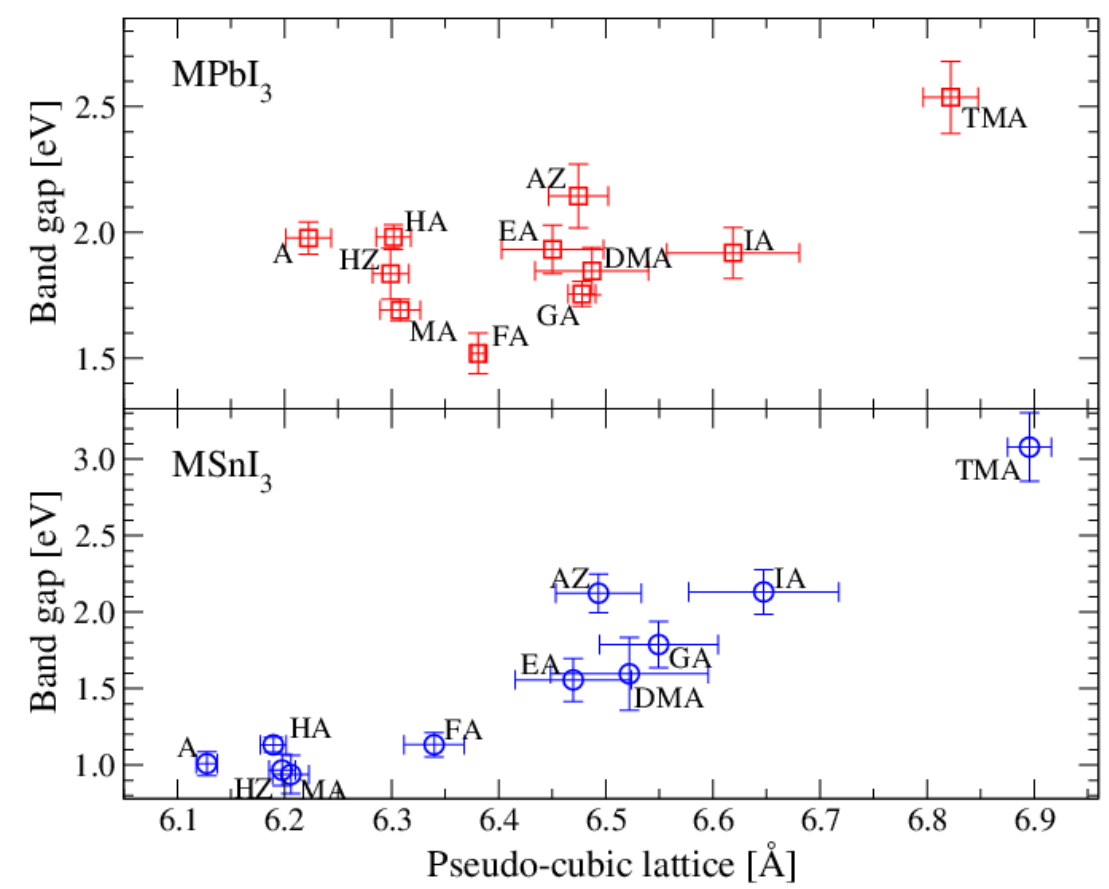

Figure 6: Calculated band gaps as a function of the pseudo-cubic lattice parameters for the set of organic molecules of Table 1 . The error bars indicate the average non-cubicity (x-axis) and the variation in the gap (y-axis) depending on the different orientations of the organic molecules.

stronger coupling between lattice and molecular orientation for the large molecules. Molecular replacements can therefore be expected to not only affect the band gap but also to play a major role for the stabilization of the different symmetrybreaking phases.

\section{Conclusions and Perspectives}

The identification and design of new light absorbing materials for use in photovoltaic or photoelectrochemical devices is traditionally based on a combination of trial and error and of sophisticated chemical/physical intuition by its practitioneers. In this chapter we have tried to illustrate how screening with ab-initio 
quantum mechanical calculations can be used as a supplementary tool in this endeavor.

The screenings necessarily have to be based on computable descriptors which address key issues in the material performance. Even though such descriptors may be quite simple and only address part of the problem they can still be useful in reducing the number of possible candidate materials for a particular application. As an example take the database discussed earlier with 19000 perovskite oxides, oxynitrides, and oxyfluorides. Considering only the band gap, heat of formation, and level alignment, the number of candidate materials for one-photon water splitting was reduced to only 20 - a much more reasonable number of materials for further investigation. Explicit calculation of the absorption efficiency could then reveal that the oxynitrides had superior absorption properties compared to the oxides.

The calculations can also be used to discover or quantify trends. For example the band gaps of the hybrid halide peroskites showed a clear correlation with the electronegativity of the anions underpinning the possibility of band gap tuning by anion substitution. Likewise the band gaps are seen to increase systematically with the size of the molecules used as A-cations, opening up for enlargement of the band gaps of Sn-based perovskites bringing them into a range of higher relevance to water splitting.

The computational exploration of perovskites as PV or PEC materials is far from complete. Both with respect to composition, crystal phases, and derived structures much territory is unexplored. Furthermore the descriptors discussed here only address very basic issues while many intersting and possibly relevant properties like electric polarization and magnetic behavior has not been addressed. 


\section{Acknowledgements}

The authors acknowledge support from the Catalysis for Sustainable Energy (CASE) initiative funded by the Danish Ministry of Science, Technology and Innovation and from the Center on Nanostructuring for the Efficient Energy Conversion (CNEEC) at Stanford University, an Energy Frontier Research Center founded by the US Department of Energy, Office of Science, Office of Basic Energy Sciences under Award No. DE-SC0001060. KST acknowledges the Danish Council for Independent Research's DFF-Sapere Aude program (grant no. 11-1051390) for financial support.

\section{References}

[1] P Hohenberg and W Kohn. Inhomogeneous Electron Gas. Physical Review, 136(3):864-871, November 1964.

[2] W Kohn and L J Sham. Self-Consistent Equations Including Exchange and Correlation Effects. Physical Review, 140(4):1133-1138, November 1965.

[3] Li-Chiang Lin, Adam H. Berger, Richard L. Martin, Jihan Kim, Kuldeep Swisher, Joseph A.and Jariwala, Chris H. Rycroft, Abhoyjit S. Bhown, Michael W. Deem, Maciej Haranczyk, and Berend Smit. In silico screening of carbon-capture materials. Nat. Mater., 11(7):633-641, 2012.

[4] G Ceder, Y-M Chiang, D R Sadoway, M K Aydinol, Y-I Jang, and B Huang. Identification of cathode materials for lithium batteries guided by firstprinciples calculations. Nature, 392:694, 1998.

[5] Stefano Curtarolo, Wahyu Setyawan, Gus L.W. Hart, Michal Jahnatek, Roman V. Chepulskii, Richard H. Taylor, Shidong Wang, Junkai Xue, Kesong Yang, Ohad Levy, Michael J. Mehl, Harold T. Stokes, Denis O. Demchenko, 
and Dane Morgan. Aflow: An automatic framework for high-throughput materials discovery. Computational Materials Science, 58:218 - 226, 2012.

[6] Geoffroy Hautier, Anna Miglio, Gerbrand Ceder, Gian-Marco Rignanese, and Xavier Gonze. Identification and design principles of low hole effective mass p-type transparent conducting oxides. Nat. Commun., 4:2292, 2013.

[7] Kristian B. Ørnsø, Juan M. García-Lastra, and Kristian S. Thygesen. Computational screening of functionalized zinc porphyrins for dye sensitized solar cells. Phys. Chem. Chem. Phys., 15:19478-19486, 2013.

[8] Mayeul d'Avezac, Jun-Wei Luo, Thomas Chanier, and Alex Zunger. Genetic-algorithm discovery of a direct-gap and optically allowed superstructure from indirect-gap si and ge semiconductors. Phys. Rev. Lett., 108:027401, 2012.

[9] Johannes Hachmann, Roberto Olivares-Amaya, Sule Atahan-Evrenk, Carlos Amador-Bedolla, Roel S. Sánchez-Carrera, Aryeh Gold-Parker, Leslie Vogt, Anna M. Brockway, and Alán Aspuru-Guzik. The harvard clean energy project: Large-scale computational screening and design of organic photovoltaics on the world community grid. The Journal of Physical Chemistry Letters, 2(17):2241-2251, 2011.

[10] Ivano E. Castelli, Thomas Olsen, Soumendu Datta, David D. Landis, Soren Dahl, Kristian S. Thygesen, and Karsten W. Jacobsen. Computational screening of perovskite metal oxides for optimal solar light capture. Energy Environ. Sci., 5:5814-5819, 2012.

[11] Ivano E. Castelli, David D. Landis, Kristian S. Thygesen, Soren Dahl, Ib Chorkendorff, Thomas F. Jaramillo, and Karsten W. Jacobsen. New cubic perovskites for one- and two-photon water splitting using the computational materials repository. Energy Environ. Sci., 5:9034-9043, 2012. 
[12] Yabi Wu, Predrag Lazic, Geoffroy Hautier, Kristin Persson, and Gerbrand Ceder. First principles high throughput screening of oxynitrides for watersplitting photocatalysts. Energy Environ. Sci., 6:157-168, 2013.

[13] Stefano Curtarolo, Gus L. W. Hart, Marco Buongiorno Nardelli, Natalio Mingo, Stefano Sanvito, and Ohad Levy. The high-throughput highway to computational materials design. Nat. Mater., 12:191-201, 2013.

[14] J. Rossmeisl, A. Logadottir, and J. K. Nørskov. Electrolysis of water on (oxidized) metal surfaces. Chemical Physics, 319(1-3):178 - 184, 2005.

[15] J. J. Mortensen, L. B. Hansen, and K. W. Jacobsen. Real-space grid implementation of the projector augmented wave method. Phys. Rev. B, 71:035109, 2005.

[16] J Enkovaara, C Rostgaard, J J Mortensen, J Chen, M Dułak, L Ferrighi, J Gavnholt, C Glinsvad, V Haikola, H A Hansen, H H Kristoffersen, M Kuisma, A H Larsen, L Lehtovaara, M Ljungberg, O Lopez-Acevedo, P G Moses, J Ojanen, T Olsen, V Petzold, N A Romero, J Stausholm-Møller, M Strange, G A Tritsaris, M Vanin, M Walter, B Hammer, H Häkkinen, G K H Madsen, R M Nieminen, J K Nørskov, M Puska, T T Rantala, J Schiøtz, K S Thygesen, and K W Jacobsen. Electronic structure calculations with gpaw: a real-space implementation of the projector augmentedwave method. Journal of Physics: Condensed Matter, 22(25):253202, 2010.

[17] Computational Materials Repository. https://cmr.fysik.dtu.dk/.

[18] Michael G Walter, Emily L Warren, James R McKone, Shannon W Boettcher, Qixi Mi, Elizabeth A Santori, and Nathan S Lewis. Solar water splitting cells. Chemical reviews, 110(11):6446-6473, November 2010.

[19] ICSDWeb. http://www.fiz-karlsruhe.de/icsd_web.html. 
[20] Materials Project - A Materials Genome Approach. http://materialsproject.org/.

[21] Bjørk Hammer, L B Hansen, and J K Nørskov. Improved adsorption energetics within density-functional theory using revised Perdew-BurkeErnzerhof functionals. Phys Rev B, 59(1):7413-7421, 1999.

[22] Kristin A. Persson, Bryn Waldwick, Predrag Lazic, and Gerbrand Ceder. Prediction of solid-aqueous equilibria: Scheme to combine first-principles calculations of solids with experimental aqueous states. Phys. Rev. B, $85: 235438,2012$.

[23] Ivano E. Castelli, Kristian S. Thygesen, and Karsten W. Jacobsen. Calculated pourbaix diagrams of cubic perovskites for water splitting: Stability against corrosion. Topics in Catalysis, 57(1):265-272, 2014.

[24] R. W. Godby, M. Schlüter, and L. J. Sham. Accurate exchange-correlation potential for silicon and its discontinuity on addition of an electron. Phys. Rev. Lett., 56:2415-2418, 1986.

[25] J. P. Perdew and Alex Zunger. Self-interaction correction to densityfunctional approximations for many-electron systems. Phys. Rev. B, 23:5048-5079, 1981.

[26] Oleg Gritsenko, Robert van Leeuwen, Erik van Lenthe, and Evert Jan Baerends. Self-consistent approximation to the kohn-sham exchange potential. Phys. Rev. A, 51:1944-1954, 1995.

[27] M. Kuisma, J. Ojanen, J. Enkovaara, and T. T. Rantala. Kohn-sham potential with discontinuity for band gap materials. Phys. Rev. B, 82:115106, 2010. 
[28] J. B. Krieger, Yan Li, and G. J. Iafrate. Construction and application of an accurate local spin-polarized kohn-sham potential with integer discontinuity: Exchange-only theory. Phys. Rev. A, 45:101-126, 1992.

[29] J. B. Krieger, Yan Li, and G. J. Iafrate. Systematic approximations to the optimized effective potential: Application to orbital-density-functional theory. Phys. Rev. A, 46:5453-5458, 1992.

[30] James D. Talman and William F. Shadwick. Optimized effective atomic central potential. Phys. Rev. A, 14:36-40, 1976.

[31] Falco Hüser, Thomas Olsen, and Kristian S. Thygesen. Quasiparticle gw calculations for solids, molecules, and two-dimensional materials. Phys. Rev. B, 87:235132, 2013.

[32] Ivano E. Castelli, Juan María García-Lastra, Falco Hüser, Kristian S Thygesen, and Karsten W Jacobsen. Stability and bandgaps of layered perovskites for one- and two-photon water splitting. New Journal of Physics, 15(10):105026, 2013.

[33] M. Shishkin and G. Kresse. Self-consistent $g w$ calculations for semiconductors and insulators. Phys. Rev. B, 75:235102, Jun 2007.

[34] Jochen Heyd, Gustavo E. Scuseria, and Matthias Ernzerhof. Hybrid functionals based on a screened coulomb potential. The Journal of Chemical Physics, 118(18):8207-8215, 2003.

[35] Ivano E. Castelli, Falco Hüser, Mohnish Pandey, Hong Li, Kristian S. Thygesen, Brian Seger, Anubhav Jain, Kristin A. Persson, Gerbrand Ceder, and Karsten W. Jacobsen. New light-harvesting materials using accurate and efficient bandgap calculations. Advanced Energy Materials, $5(2): 1400915,2015$. 
[36] Jun Yan, Jens. J. Mortensen, Karsten W. Jacobsen, and Kristian S. Thygesen. Linear density response function in the projector augmented wave method: Applications to solids, surfaces, and interfaces. Phys. Rev. B, 83:245122, 2011.

[37] Liping Yu and Alex Zunger. Identification of potential photovoltaic absorbers based on first-principles spectroscopic screening of materials. Phys. Rev. Lett., 108:068701, 2012.

[38] M. A. Butler and D. S. Ginley. Prediction of flatband potentials at semiconductor-electrolyte interfaces from atomic electronegativities. Journal of The Electrochemical Society, 125(2):228-232, 1978.

[39] Yong Xu and Martin A.A. Schoonen. The absolute energy positions of conduction and valence bands of selected semiconducting minerals. American Mineralogist, 85(3-4):543-556, 2000.

[40] Mihai V. Putz, Nino Russo, and Emilia Sicilia. About the mulliken electronegativity in dft. Theor. Chem. Acc., 114(1-3):38-45, 2005.

[41] Yabi Wu, M Chan, and G Ceder. Prediction of semiconductor band edge positions in aqueous environments from first principles. Physical Review B, 83(23):235301, June 2011.

[42] Vladan Stevanovic, Stephen Lany, David S Ginley, William Tumas, and Alex ZUNGER. Assessing Capability of Semiconductors to Split Water Using Ionization Potentials and Electron Affinities Only. Physical Chemistry Chemical Physics, 2013.

[43] Peter C K Vesborg and Thomas F Jaramillo. Addressing the terawatt challenge: Scalability in the supply of chemical elements for renewable energy. RSC Advances, 2012. 
[44] Tatsumi Ishihara. Perovskite Oxide for Solid Oxide Fuel Cells. Springer Verlag, June 2009.

[45] Rosiana Aguiar, Dmitry Logvinovich, Anke Weidenkaff, Anita Rachel, Armin Reller, and Stefan G. Ebbinghaus. The vast colour spectrum of ternary metal oxynitride pigments. Dyes and Pigments, 76(1):70 - 75, 2008.

[46] I E Castelli and K W Jacobsen. Designing rules and probabilistic weighting for fast materials discovery in the perovskite structure. Modelling and Simulation in Materials Science and Engineering, 22(5):055007, 2014.

[47] S. Trasatti. Croat. Chem. Acta, 63:313-329, 1990.

[48] M.F. Weber and M.J. Dignam. Splitting water with semiconducting photoelectrodes-efficiency considerations. International Journal of Hydrogen Energy, 11(4):225 - 232, 1986.

[49] Weifeng Zhang, Junwang Tang, and Jinhua Ye. Structural, photocatalytic, and photophysical properties of perovskite $\operatorname{msno} 3(\mathrm{~m}=\mathrm{ca}$, sr, and ba) photocatalysts. Journal of Materials Research, 22:1859-1871, 2007.

[50] Hideki Kato, Hisayoshi Kobayashi, and Akihiko Kudo. Role of ag+ in the band structures and photocatalytic properties of agmo3 (m: Ta and nb) with the perovskite structure. The Journal of Physical Chemistry B, 106(48):12441-12447, 2002.

[51] Daisuke Yamasita, Tsuyoshi Takata, Michikazu Hara, Junko N. Kondo, and Kazunari Domen. Recent progress of visible-light-driven heterogeneous photocatalysts for overall water splitting. Solid State Ionics, 172(1-4):591 - 595, 2004. Proceedings of the Fifteenth International Symposium on the Reactivity of Solids. 
[52] Ivano E. Castelli, Kristian S. Thygesen, and Karsten W. Jacobsen. Calculated optical absorption of different perovskite phases. J. Mater. Chem. A, $3: 12343-12349,2015$.

[53] Ivano E. Castelli, Kristian S. Thygesen, and Karsten W. Jacobsen. Bandgap engineering of double perovskites for one- and two-photon water splitting. In Symposium QQ - Materials Informatics, volume 1523 of MRS Proceedings, 2013.

[54] Akihiro Kojima, Kenjiro Teshima, Yasuo Shirai, and Tsutomu Miyasaka. Organometal halide perovskites as visible-light sensitizers for photovoltaic cells. Journal of the American Chemical Society, 131(17):6050-6051, May 2009.

[55] Nam Joong Jeon, Jaemin Lee, Jun Hong Noh, Mohammad Khaja Nazeeruddin, Michael GrÃd’tzel, and Sang Il Seok. Efficient inorganicâĂŞorganic hybrid perovskite solar cells based on pyrene arylamine derivatives as hole-transporting materials. Journal of the American Chemical Society, 135(51):19087-19090, 2013.

[56] Jeffrey A. Christians, Raymond C. M. Fung, and Prashant V. Kamat. An inorganic hole conductor for organo-lead halide perovskite solar cells. improved hole conductivity with copper iodide. Journal of the American Chemical Society, 136(2):758-764, 2014.

[57] Eran Edri, Saar Kirmayer, Michael Kulbak, Gary Hodes, and David Cahen. Chloride inclusion and hole transport material doping to improve methyl ammonium lead bromide perovskite-based high open-circuit voltage solar cells. The Journal of Physical Chemistry Letters, 5(3):429-433, 2014. 
[58] Surya Prakash Singh and P. Nagarjuna. Organometal halide perovskites as useful materials in sensitized solar cells. Dalton Trans., 43:5247-5251, 2014.

[59] Ivano E. Castelli, Juan María García-Lastra, Kristian S. Thygesen, and Karsten W. Jacobsen. Bandgap calculations and trends of organometal halide perovskites. APL Materials, 2(8):081514, 2014.

[60] John P. Perdew, Adrienn Ruzsinszky, Gábor I. Csonka, Oleg A. Vydrov, Gustavo E. Scuseria, Lucian A. Constantin, Xiaolan Zhou, and Kieron Burke. Restoring the density-gradient expansion for exchange in solids and surfaces. Phys. Rev. Lett., 100:136406, 2008.

[61] D. Usanmaz, P. Nath, J. J. Plata, G. L. W. Hart, I. Takeuchi, M. Buongiorno Nardelli, M. Fornari, and S. Curtarolo. First principles thermodynamical modeling of the binodal and spinodal curves in lead chalcogenides. ArXiv e-prints, 1509.00502, September 2015.

[62] David E. Scaife, Paul F. Weller, and Wayne G. Fisher. Crystal preparation and properties of cesium tin(ii) trihalides. Journal of Solid State Chemistry, 9(3):308 - 314, 1974.

[63] Paolo Umari, Edoardo Mosconi, and Filippo De Angelis. Relativistic gw calculations on ch3nh3pbi3 and ch3nh3sni3 perovskites for solar cell applications. Sci. Rep., 4(4467), 2014.

[64] Anna Amat, Edoardo Mosconi, Enrico Ronca, Claudio Quarti, Paolo Umari, M. K. Nazeeruddin, Michael Grätzel, and Filippo De Angelis. Cation-induced band-gap tuning in organohalide perovskites: Interplay of spin-orbit coupling and octahedra tilting. Nano Letters, 14(6):3608-3616, 2014. 
[65] Ivo Borriello, Giovanni Cantele, and Domenico Ninno. Ab initio investigation of hybrid organic-inorganic perovskites based on tin halides. Phys. Rev. B, 77:235214, 2008.

[66] Hong Li, Ivano Eligio Castelli, Kristian Sommer Thygesen, and Karsten Wedel Jacobsen. Strain sensitivity of band gaps of Sn-containing semiconductors. Physical Review B, 91(4):045204, January 2015.

[67] Gregor Kieslich, Shijing Sun, and Anthony K. Cheetham. Solid-state principles applied to organic-inorganic perovskites: new tricks for an old dog. Chem. Sci., 5:4712-4715, 2014.

[68] Jarvist M Frost, Keith T Butler, Federico Brivio, Christopher H Hendon, Mark van Schilfgaarde, and Aron Walsh. Atomistic Origins of High-Performance in Hybrid Halide Perovskite Solar Cells. Nano Letters, 14(5):2584-2590, May 2014. 